\title{
Reducing Sensing Error in Cognitive PANs Through Differential Sensing
}

\author{
Jelena Mišić* \\ University of Manitoba \\ Winnipeg MB, Canada \\ jmisic@cs.umanitoba.ca
}

\author{
Vojislav B. Mišić \\ University of Manitoba \\ Winnipeg MB, Canada \\ vmisic@cs.umanitoba.ca
}

\begin{abstract}
Cognitive radio technology necessitates accurate and timely sensing of the primary users' activity on the chosen set of channels. If sensing error is unacceptably high, we can undertake differential sensing in which subsets of sensing nodes target idle and active channels, respectively, or even reduce the number of working channels so as to improve the channels-vs.-sensing nodes ratio. The paper presents a probabilistic analysis of a flexible differential sensing policy and investigates the range of values in which such, incomplete sensing is capable of maintaining an accurate view of the status of the working channel set.
\end{abstract}

\section{Categories and Subject Descriptors}

C.2 [Computer-Communication Networks]: C.2.1 Network Architecture and Design-Wireless communication; C.4 [Performance of Systems]: Modeling Techniques, Performance Attributes

\section{General Terms}

Algorithms, Performance

\section{Keywords}

cognitive communications, wireless PANs, spectrum sensing, differential sensing

\section{INTRODUCTION}

Cognitive communications technology [1] is predominantly envisaged for use in the area of local and wide-range networks [3, 6], but it can benefit wireless personal area networks every bit as well [4]. Opportunistic spectrum access and co-existence of several WPANs in the same physical area may be achieved through frequency hopping [8] in which the channel time is slotted into superframes of suitable size, possibly divided into specialized slots as in, e.g., [16]. While this approach is similar to the one used in

\footnotetext{
${ }^{*}$ This research has been supported by an NSERC Discovery Grant.
}

Permission to make digital or hard copies of all or part of this work for personal or classroom use is granted without fee provided that copies are not made or distributed for profit or commercial advantage and that copies bear this notice and the full citation on the first page. To copy otherwise, to republish, to post on servers or to redistribute to lists, requires prior specific permission and/or a fee.

QShine July 28-31, 2008, Hong Kong, China

Copyright 2008 ICST ISBN 978-963-9799-26-4

DOI 10.4108/ICST.QSHINE2008.3885
Bluetooth [2], the hopping sequence of a PAN with cognitive access (cognitive PAN, or CPAN) should dynamically adapt to the current activity patterns on the working channel set. In this manner, the interference both to and from primary users will be can be minimized, and CPAN users can enjoy the full benefits of both frequency hopping and opportunistic access paradigms.

Dynamic determination of the hopping sequence is based on timely information about primary users' activity, which must be obtained by sensing. Unfortunately, sensing incurs errors, which may be partially offset by performing sensing in regular intervals [15] and in a cooperative manner by several among the CPAN nodes [17], but the manner of selection of channels to be sensed remains the most critical issue. Random selection of channels with equal probability (hereafter referred to as uniform random sensing) ensures that all channels are sensed with equal frequency and with the same error in statistical sense [12]. However, not all errors affect the operation of a CPAN in the same way: a channel that becomes idle while considered active is but a missed opportunity, whereas a channel that becomes active during CPAN operation may cause interference and, in extreme cases, complete failure of communication in the CPAN.

Therefore, the onset of primary user activity on an idle channel is more critical for the CPAN operation, and should be sensed with smaller error than the preceding (or succeeding) end of such activity. Smaller error for idle channels requires shorter sensing intervals, which may be accomplished by partitioning the set of sensing nodes into semi-specialized subsets, as will be explained below. The performance of this technique is modeled and analyzed through a queueing theoretic approach, and compared to that of uniform random sensing. The differential sensing approach is shown to be capable of reducing the error in both magnitude and duration; in cases where this reduction is deemed insufficient, the number of working channels may be reduced to improve the ratio of channels to sensing nodes.

The paper is organized as follows: Section 2 gives more details about the operation of a frequency hopping cognitive personal area network and the sensing process in particular, together with a detailed description of the channel sensing policies under consideration. Section 3 presents the probabilistic model of the sensing activity, while Section 4 models probabilities of incorrect channel information. Section 5 presents and discusses performance of these policies. Finally, Section 6 concludes the paper and highlights some avenues for future research.

\section{CPAN OPERATION AND SENSING}

Let us consider a generic CPAN piconet, a small network consisting of a dedicated coordinator and a number of nodes. We assume that the available time is partitioned into slots or superframes 
of fixed size, similar to IEEE 802.15.3 [7], which may further be subdivided in to a number of specialized subframes. Each superframe is marked by a beacon frame emitted by the coordinator, and each superframe uses a different RF channel from the working frequency band. All devices in the piconet use a frequency hopping spread spectrum approach to multiple access; the hopping sequence is not only random (insofar as technically possible) but also adaptive, in the sense that any channel currently occupied by a primary user ought to be avoided.

One of the main functions of the piconet coordinator is to maintain an accurate and timely channel map, i.e., the map of primary user activity on each of the $N$ working channels. In each superframe the coordinator will instruct $X$ secondary users to sense and report back the status of $X$ distinct channels. Upon receiving this information, the coordinator updates the channel map, selects the best channel for a subsequent frequency hop, and announces it to the members of its piconet. All communications related to piconet administration, including sensing, take place on a dedicated control channel not used by any primary user.

The number of sensing nodes $X$ should ideally be equal to, or larger than, the number of channels $N$ in the working set, so that the channel map may be kept accurate. As sensing is performed in discrete intervals, any change in the state of a channel can be detected only upon the next sensing event; therefore, state changes in the channel map are always delayed with respect to the actual changes.

When there are more channels than sensing nodes, i.e., $N>X$, not every channel can be sensed in every sensing cycle, and the information in the channel map is only partially correct at any given time. The magnitude of this error will determine the success rate of CPAN transmissions and, ultimately, its quality of service. The error may be expressed through the mean number of channels with incorrect information and the delay in detecting changes in channel status. Error reduction may be accomplished by increasing the number of sensors or by reducing the number of working channels, whichever is more convenient.

Not all errors have the same impact, though. A missed idle channel is just a missed opportunity which is not too critical as long as there are other idle channels available; a missed activity on a channel, on the other hand, can harm the CPAN operation if that channel has been selected for data transmission. In order to reduce the probability of errors of the latter kind, the delay in detecting the beginning of primary user activity (i.e., the end of a spectral opportunity) should be lower than the corresponding delay in detecting its end (i.e., the beginning of a spectral opportunity). This may be accomplished by sensing idle channels more frequently than the active ones. Note that, in this context, 'idle' refers to channels which are marked as idle in the channel map, since this is the only information available to a CPAN piconet and its coordinator.

If the selection of channels to be sensed is performed in a pseudorandom manner with uniform distribution of targets, each channel will be sensed with equal probability, and mean sensing intervals will be the same regardless of channel state. Preferential treatment of idle channels may be accomplished by differential sensing, i.e., partitioning the set of $X$ sensing nodes into distinct subsets with $X_{i}<X$ and $X_{a}=X-X_{i}$ members, respectively. The sensors from the first subset, hereafter referred to as guard sensors, are assigned to idle channels; the sensors from the second subset are assigned to active channels. The subset membership may or may not be fixed; what is important is that each subset has a specified number of sensing nodes in each sensing cycle. By adjusting the value of $X_{i}$, we can reduce or increase the mean sensing interval within certain limits and, thus, reduce the sensing error for idle channels (the corresponding error for active channels will be changed in inverse proportion).

Sometimes the number of idle channels may be smaller than $X_{i}$, or the number of active channels is smaller than $X_{a}$. Rather than leaving such 'extra' sensors out of the sensing process, we propose to add them temporarily to the other subset, and thus utilize the full number of $X$ sensing nodes in each sensing cycle.

\section{ANALYTICAL MODEL OF DIFFEREN- TIAL SENSING}

Let us now present a queueing theoretic model of differential sensing process. Let the working spectrum band be partitioned into $N$ narrowband RF channels (which we will refer to as the working channel set), and let each primary user be active on a distinct channel from that group. (The common control channel is excluded from this group.) Let us also denote relevant variables in the active and idle periods of the channel state, with subscripts $a$ and $i$, respectively, while subscripts $r$ and $o$ refer to real and observed values of the network parameters, respectively. In the analysis that follows, we assume that active and idle times on each channel follow probability distributions with cumulative density functions $T_{a, r}(x)$ and $T_{i, r}(x)$, and mean values of $\overline{T_{a, r}}$ and $\overline{T_{i, r}}$, respectively. Actual mean value of the cycle time will be denoted as $\overline{T_{c y c, r}}=\overline{T_{a, r}}+\overline{T_{i, r}}$.

Each sensing node performs sensing in a frequency hopping manner with the normalized sensing period of $T_{s}$; for convenience, we set $T_{s}=1$. Assuming that $T_{s} \ll \overline{T_{a}}, \overline{T_{i}}$, the probability distributions of active and idle times of primary users may be taken to be discrete, and their state changes can occur at the boundaries of the sensing period $T_{s}$. Let $a$ and $i$ be discrete random variables taking values on some subset of the non-negative integers. Then, the Probability Generating Functions (PGFs) for $a$ and $i$ can be defined as $T_{a}(z)=\sum_{k=0}^{\infty} p_{a}(k) z^{k}$ and $T_{i}(z)=\sum_{k=0}^{\infty} p_{i}(k) z^{k}$, where $p_{a}$ and $p_{i}$ are the probability mass functions of $a$ and $i$, respectively [14]. For example, if $T_{a, r}$ and $T_{i, r}$ are geometrically distributed with parameters $\alpha$ and $\beta$ respectively, then $p_{a}(k)=\alpha(1-\alpha)^{k-1}$ and $p_{i}(k)=\beta(1-\beta)^{k-1}$.

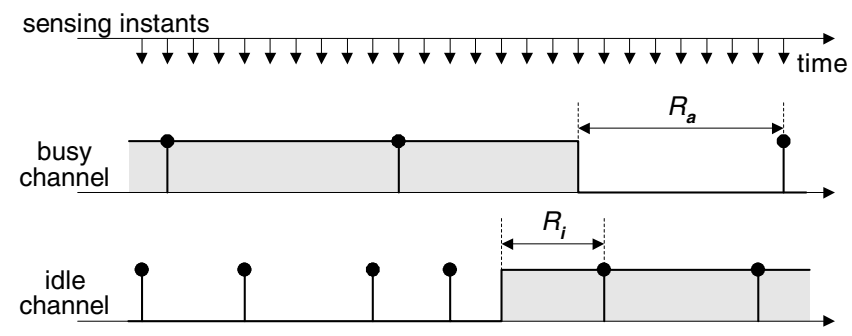

Figure 1: Delays in detecting the beginning and the end of spectral opportunities under differential sensing.

\subsection{Sensing periods and residual sensing times}

Let the mean observed durations of active and inactive period be $\overline{T_{i, o}}$ and $\overline{T_{a, o}}$, respectively. Fig. 1 shows the relationship of actual and observed (in)activity period on a given channel, together with the associated detection delays. Then, the probability that a channel is observed to be idle is $p_{i, o}=\overline{T_{i, o}} /\left(\overline{T_{a, o}}+\overline{T_{i, o}}\right)$, and the observed probability that channel is occupied by the primary user is $p_{a, o}=1-p_{i, o}$. The mean observed numbers of idle and active channels are $\overline{N_{i, o}}=p_{i, o} N$ and $\overline{N_{a, o}}=p_{a, o} N=N-\overline{N_{i, o}}$, 
respectively. The probability that a channel is actually idle is $p_{i, r}=$ $\overline{T_{i, r}} /\left(\overline{T_{a, r}}+\overline{T_{i, r}}\right) \neq p_{i, o}$; by the same token, $p_{a, r} \neq p_{a, o}$.

The choice of channels to be sensed is made in a random fashion within each group-idle and active nodes - but no channel is assigned to two or more sensing nodes in any given sensing cycle. The probability of selecting idle or active channel from the non-guard group is $p_{i}=\left(\overline{N_{i, o}}-X_{i}\right) / X_{a}$ and $p_{a}=\overline{N_{a, o}} / X_{a}$, respectively. Let us denote the probabilities that the target idle or active channel will be sensed in one sensing cycle as $P_{w, i}$ and $P_{w, a}$, respectively; their values are

$$
\begin{aligned}
& P_{w, i}= \begin{cases}\sum_{k=1}^{m_{i}}\left(\begin{array}{c}
X_{a} \\
k
\end{array}\right) p_{i}^{k}\left(1-p_{i}\right)^{X_{a}-k} \frac{k+X_{i}}{\overline{N_{i, o}}}, & X_{i}<\overline{N_{i, o}} \\
1, & X_{i} \geq \overline{N_{i, o}}\end{cases} \\
& P_{w, a}= \begin{cases}\sum_{a}^{m_{a}}\left(\begin{array}{c}
X_{a} \\
k
\end{array}\right) p_{a}^{k}\left(1-p_{a}\right)^{X_{a}-k} \frac{k}{\overline{N_{a, o}}}, & X_{i}<\overline{N_{i, o}} \\
\frac{X-\overline{N_{i, o}}}{\overline{N_{a, o}}}, & X_{i} \geq \overline{N_{i, o}}\end{cases}
\end{aligned}
$$

The time between two consecutive sensing events has a geometric distribution. As probabilities of selecting idle and active channels differ, so do the appropriate PGFs, which are

$$
\begin{aligned}
& B_{i}(z)= \begin{cases}\sum_{i=1}^{\infty} P_{w, i}\left(1-P_{w, i}\right)^{i-1} z^{i}=\sum_{j=1}^{\infty} b_{i, j} z^{j}, & X_{i}<\overline{N_{i, o}} \\
z, & X_{i} \geq \overline{N_{i, o}}\end{cases} \\
& B_{a}(z)= \begin{cases}\sum_{i=1}^{\infty} P_{w, a}\left(1-P_{w, a}\right)^{i-1} z^{i}=\sum_{j=1}^{\infty} b_{a, j} z^{j}, & X_{a}<\overline{N_{a, o}} \\
z, & X_{a} \geq \overline{N_{a, o}}\end{cases}
\end{aligned}
$$

for idle and active channels, respectively. The mean values of these times are $\overline{b_{i}}=\lim _{z \rightarrow 1} \frac{\partial B_{i}(z)}{\partial z}=B_{i}^{\prime}(1)$, for idle channels, and $\overline{b_{a}}=$ $\lim _{z \rightarrow 1} \frac{\partial B_{a}(z)}{\partial z}=B_{a}^{\prime}(1)$, for active ones.

Next, we need to find the time between the actual change of channel state and the moment when it is detected through sensing. The sensing process for a single channel may be considered as a discrete-time renewal process where sensing events correspond to renewal points, while the renewal time corresponds to the period between two consecutive sensing events [5]. In terms of renewal theory, the time between the channel state change and the next sensing event is denoted as the residual life (time) or forward recurrence time; note that the channel state change can occur anywhere between two sensing points (which take place on the basic sensing slot boundary). This residual time defines the time period in which the where information about the channel state might differ from the actual channel state. Let us consider a transition from idle to active state, and a random edge $j$ of the sensing slot within a sensing period. The probability that the next sensing event will occur exactly $k$ basic sensing slots later is $b_{i, j+k} / \overline{b_{i}}$. By summing over all basic sensing slots within the sensing period, the probability that the residual sensing time is $k$ basic sensing slots is obtained as

$$
R_{i, k}=\sum_{j=1}^{\infty} \frac{b_{i, j+k}}{\overline{b_{i}}}=\sum_{j=k+1}^{\infty} \frac{b_{i, j}}{\overline{b_{i}}}
$$

Note that the first subscript of $R_{i, k}$ denotes that the channel is idle, whereas the second one corresponds to the index within the sequence.
Then, the PGF for the residual sensing time is

$$
R_{i}(z)=\sum_{k=0}^{\infty} R_{i, k} z^{k}=\frac{1-B_{i}(z)}{\overline{b_{i}}(1-z)}
$$

while its mean value is

$$
\overline{R_{i}}=B_{i}^{\prime \prime}(1) /\left(2 B_{i}^{\prime}(1)\right)
$$

where $B_{i}^{\prime \prime}(1)=\lim _{z \rightarrow 1} \frac{\partial^{2} B_{i}(z)}{\partial z^{2}}$. In a similar fashion, we can derive the PGF for the distribution of the residual sensing time for activeto-idle transitions and its mean value as

$$
\begin{aligned}
& R_{a}(z)=\sum_{k=0}^{\infty} R_{a, k} z^{k}=\frac{1-B_{a}(z)}{\overline{b_{a}}(1-z)} \\
& \overline{R_{a}}=B_{a}^{\prime \prime}(1) /\left(2 B_{a}^{\prime}(1)\right)
\end{aligned}
$$

\subsection{Observed durations of inactive and active periods}

We are now ready to find the probability distribution of the observed duration of active and inactive period of a channel. As noted before, the observed durations of idle and active channel periods will be affected by delays in detecting the channel state changes. In extreme situations where residual sensing time is larger than the period under observation (idle or active), an entire (in)activity period may be skipped. Given the PGFs for residual sensing times, the probability that an entire period is skipped is

$$
\begin{aligned}
& p_{s, i}=\sum_{k=2}^{\infty} R_{a, k} \sum_{n=1}^{k-1} p_{i}(n)=\sum_{n=1}^{\infty} p_{i}(n) \sum_{k=n+1}^{\infty} R_{a, k} \\
& p_{s, a}=\sum_{k=2}^{\infty} R_{i, k} \sum_{n=1}^{k-1} p_{a}(n)=\sum_{n=1}^{\infty} p_{a}(n) \sum_{k=n+1}^{\infty} R_{i, k}
\end{aligned}
$$

where the second form in both equations has been obtained from the first by exchanging the order of summation of series.

We can now define the probability distributions of the duration of idle and active periods conditioned on the event that they are not skipped by the sensing, i.e., that at least one sensing event occurs during the idle or active period:

$$
\begin{aligned}
\Theta_{i}(z)\left(1-p_{s, i}\right) & =\sum_{n=1}^{\infty} p_{i}(n) z^{n} \sum_{k=0}^{n} R_{a, k} \\
\Theta_{a}(z)\left(1-p_{s, a}\right) & =\sum_{n=1}^{\infty} p_{a}(n) z^{n} \sum_{k=0}^{n} R_{i, k}
\end{aligned}
$$

From (7) and (8), it follows that $1-p_{s, i}=\sum_{n=1}^{\infty} p_{i}(n) \sum_{k=0}^{n} R_{a, k}$ and $1-p_{s, a}=\sum_{n=1}^{\infty} p_{a}(n) \sum_{k=0}^{n} R_{i, k}$. Therefore, $\Theta_{i}(1)=1$ and $\Theta_{a}(1)=1$, which shows that $\Theta_{i}(z)$ and $\Theta_{a}(z)$ are indeed probability generating functions.

Finally, we can derive the expressions for the probability distribution of observed values of idle and active periods on the channel. The PGF for the duration of idle period is

$$
\begin{aligned}
T_{i, o}(z)= & \Theta_{i}(z)\left(\left(1-p_{s, a}\right)+\left(1-p_{s a}\right) p_{s, a} z^{\overline{T_{c y c, r}}}\right. \\
& \left.+\left(1-p_{s a}\right)\left(p_{s, a} z^{\overline{T_{c y c, r}}}\right)^{2}+\ldots+\right)
\end{aligned}
$$

After summing the infinite series we obtain

$$
T_{i, o}(z)=\frac{\Theta_{i}(z)\left(1-p_{s, a}\right)}{1-p_{s, a} z^{T_{c y c, r}}} R_{i}(z)
$$


Since $\Theta_{i}(1)=1$, it follows that $T_{i, o}(1)=1$, which shows that $T_{i, o}(z)$ is indeed the probability generating function and its mean value is

$$
\overline{T_{i, o}}=T_{i, o}^{\prime}(1)+\overline{R_{i}}
$$

In a similar way, we obtain

$$
T_{i, a}(z)=\frac{\Theta_{a}(z)\left(1-p_{s, i}\right)}{1-p_{s, i} z^{\bar{T}_{c y c, r}}} R_{a}(z)
$$

with mean value of $\overline{T_{a, o}}=T_{a, o}^{\prime}(1)+\overline{R_{a}}$.

Equations (1), (5) (6), (8), (7), (9), (11) and (13), allow us to find the values of $p_{i, o}, p_{i, a} P_{w i}, P_{w a}, \overline{T_{i, o}}, \overline{T_{a, o}}$, and $\overline{R_{i}}, \overline{R_{a}}$. Furthermore, mean detection delays are $\left(1-p_{s, i}\right) \overline{R_{a}}$ and $\left(1-p_{s, a}\right) \overline{R_{i}}$, for detection of idle and active channel state, respectively.

\section{PROBABILITY OF INCORRECT INFOR- MATION ABOUT CHANNEL ACTIVITY}

Let us now consider one active period of a primary user. All sensing points within this period will find the channel active; this will be (correctly) recorded in the channel map. Once the primary user ends its activity, this information is rendered incorrect; it will become correct again upon the next sensing point which occurs, on the average, $\overline{R_{a}}$ basic sensing slots after the first state transition of the primary channel. If the probability that the primary user will have a single transition from active to idle state between two successive sensing events is $P_{a}^{(1)}=\sum_{n=0}^{\infty} R_{a, n} \sum_{k=n+1}^{\infty} p_{i}(k)$, the joint PGF for the duration of residual sensing time and idle time of the primary user may be expressed as

$$
G_{a}^{(1)}(z, y)=\frac{1}{P_{a}^{(1)}} \sum_{n=0}^{\infty} R_{a, n} z^{n} \sum_{k=n+1}^{\infty} p_{i}(k) y^{k}
$$

Then, the time interval during which the channel state information in the channel map is incorrectly considered active is

$$
L_{a}=P_{a}^{(1)} \frac{\partial G_{a}^{(1)}(1,1)}{\partial z}+\epsilon
$$

where $\frac{\partial G_{a}^{(1)}(1,1)}{\partial z}=\lim _{z \rightarrow 1} \lim _{y \rightarrow 1} \frac{\partial G_{a}^{(1)}(z, y)}{\partial z}$, and $\epsilon$ denotes a small error due to the possibility of incorrect information caused by multiple state transitions. Namely, in the ideal case a single sensing period (i.e., the period between two consecutive sensing events) should capture a single state change of the primary user, as shown in Fig. 2(a). However, a channel may change state more than once between two consecutive sensing events, as in Figs. 2(b) and (c); in that case, the duration of incorrect information about the channel state will differ from the residual time, which means that the latter is not useful beyond a mere first approximation to the former. The difference may easily be obtained but the detailed derivation, which can be found in [11], is not shown here due to limited space.

The probability that the channel is incorrectly observed to be in the active state is

$$
a_{1}=\frac{L_{a}}{\overline{T_{a, o}}}
$$

while the complementary probability (that the information about the channel being in the active state is correct) is $a_{0}=1-a_{1}$. The corresponding probabilities for the inactive (idle) state, $b_{1}$ and $b_{0}$, respectively, can be found as

$$
b_{1}=\frac{L_{i}}{\overline{T_{i, o}}}
$$
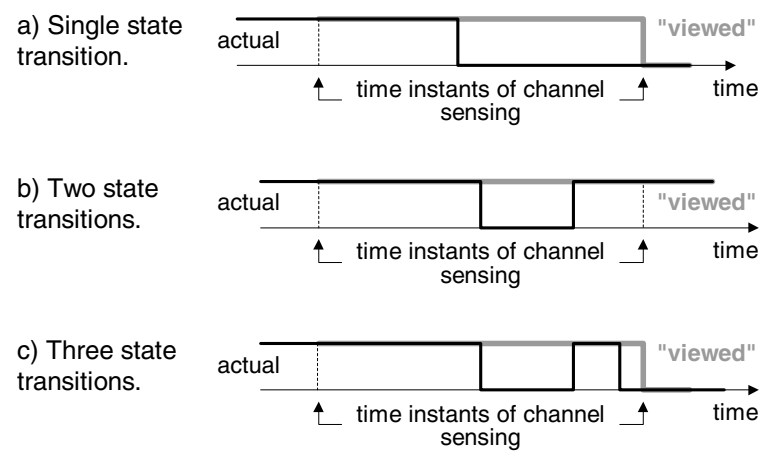

Figure 2: Timing of channel sensing and channel state change.

and $b_{0}=1-b_{1}$.

Given that the primary user is observed as idle or active with the probabilities $p_{i, o}=\overline{T_{i, o}} /\left(\overline{T_{a, o}}+\overline{T_{i, o}}\right)$ and $p_{a, o}=1-p_{i, o}$, respectively, the number of channels with obsolete information can be characterized with the PGF of

$$
\begin{gathered}
E(w, z, y)=\sum_{k=0}^{\infty} \sum_{i=0}^{k} \sum_{j=0}^{N-k}\left(\begin{array}{c}
N \\
k
\end{array}\right) p_{a, o}^{k}\left(1-p_{a, o}\right)^{N-k} w^{k} \\
\cdot\left(\begin{array}{c}
k \\
i
\end{array}\right) a_{1}^{i}\left(1-a_{1}\right)^{k-i} z^{i}\left(\begin{array}{c}
N-k \\
j
\end{array}\right) b_{1}^{j}\left(1-b_{1}\right)^{N-k-j} y^{j}
\end{gathered}
$$

Mean number of observed active and inactive channels can, then, be obtained as $N_{a, o}=\frac{\partial E(1,1,1)}{\partial w}$ and $N_{a, i}=N-N_{a, o}$, respectively. The mean number of channels for which the information of being in the active or idle state (obtained earlier) is obsolete, is $E_{a}=\frac{\partial E(1,1,1)}{\partial z}$ and $E_{i}=\frac{\partial E(1,1,1)}{\partial y}$, for active and idle channels, respectively.

\section{PERFORMANCE EVALUATION}

Let us now investigate the practical implications of the formulae derived above. To that end, let us assume that the distribution of active and idle periods of primary user activity is geometric, i.e., $T_{a, r}(z)=\sum_{k=1}^{\infty} \alpha(1-\alpha)^{k-1} z^{k}$ and $T_{i, r}(z)=\sum_{k=0}^{\infty} \beta(1-$ $\beta)^{k-1} z^{k}$, with parameters $\alpha=1 / \overline{T_{a, r}}$ and $\beta=1 / \overline{T_{i, r}}$. (Other distributions could be substituted with ease, since no change of the general framework is necessary.) Furthermore, we have set the number of channels (and primary users) to $N=25$, unless variable, and the number of sensing nodes to $X=10$, since we want to assess the performance of sensing, both uniform and differential, when the number of sensing nodes is much smaller than the number of channels. The cycle time of the primary user was set to $T_{c y c, r}=150$, while the basic sensing period is $T_{s}=1$.

A total of three different experiments were performed. First, we have applied uniform random sensing, and varied the number of primary users from $N=15$ to 50 , while the duty cycle of these users was varied between $\gamma=0.1$ to 0.9. Second, we have applied differential sensing with $X_{i}=5$, with the same independent variables. Finally, we have fixed the number of channels to $N=25$ and varied the number of nodes in the guard sensor set from $X_{i}=0$ to 8 . With these values, we have solved the equations shown above to calculate the following performance measures: mean sensing interval, mean number of channels with incorrect (obsolete) information in the channel map, and mean duration (lifetime) of that information, for both idle and active channels. Our calculations 


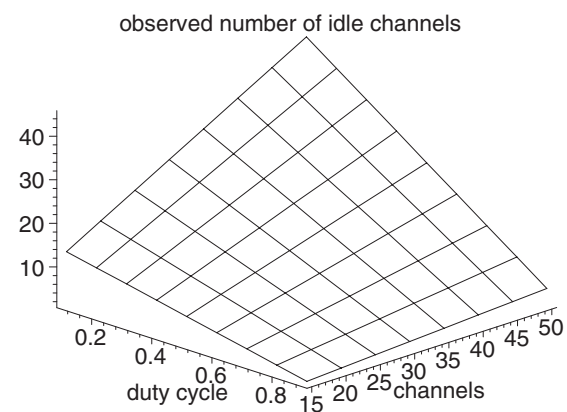

(a) Mean number of idle channels in the channel map under uniform random sensing, $N$ and $\gamma$ variable.

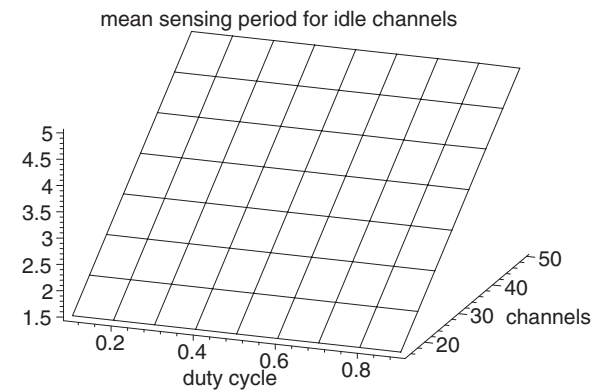

(d) Mean sensing interval for idle channels under uniform random sensing, $N$ and $\gamma$ variable.

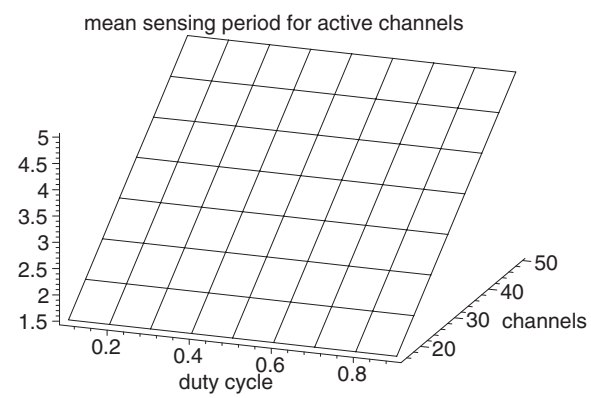

(g) Mean sensing interval for active channels under uniform random sensing, $N$ and $\gamma$ variable.

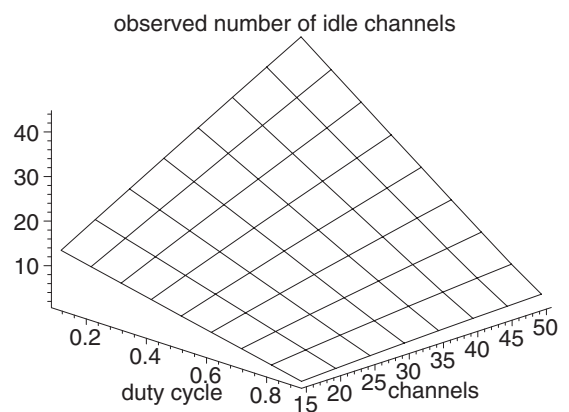

(b) Mean number of idle channels in the channel map under split sensing, $N$ and $\gamma$ variable.

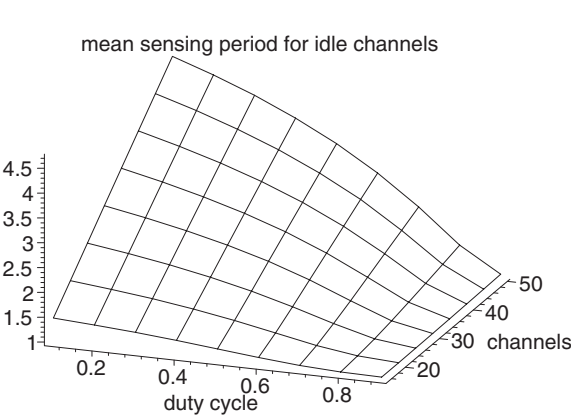

(e) Mean sensing interval for idle channels under split sensing, $N$ and $\gamma$ variable.

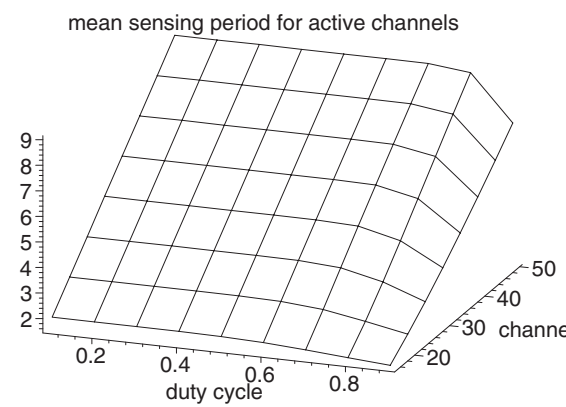

(h) Mean sensing interval for active channels under split sensing, $N$ and $\gamma$ variable.

Figure 3: Pertaining to the sensing process.

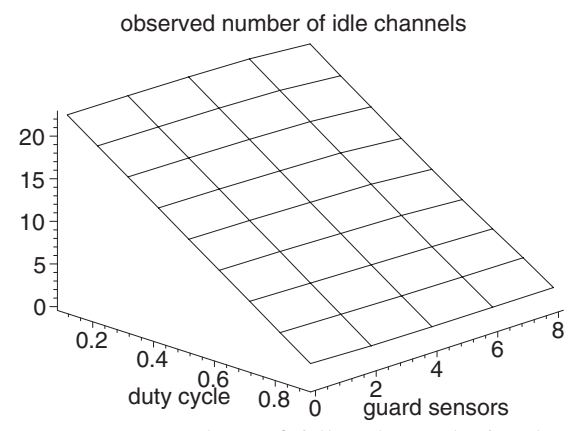

(c) Mean number of idle channels in the channel map under split sensing, $\gamma$ and $X_{i}$ variable.

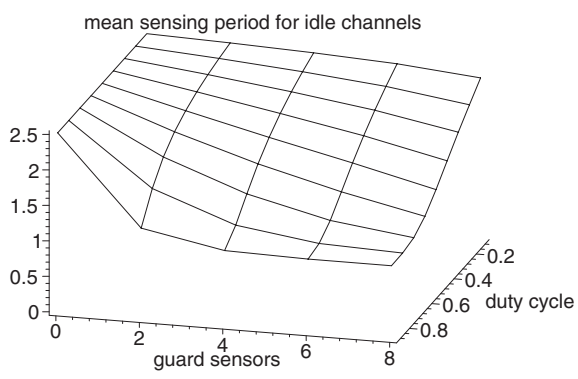

(f) Mean sensing interval for idle channels under split sensing, $\gamma$ and $X_{i}$ variable.

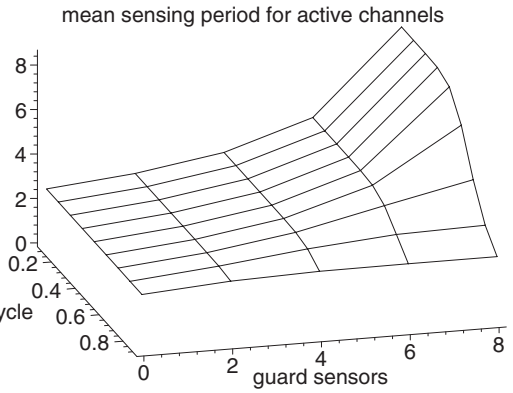

(i) Mean sensing interval for active channels under split sensing, $\gamma$ and $X_{i}$ variable. were done using Maple 11 from Maplesoft, Inc. [9], truncating the infinite series for various PGFs after 500 members.

The main parameters of the sensing process are presented in Fig. 3: top row shows the mean number of idle channels as obtained from the channel map, while the middle and bottom rows show mean sensing intervals for idle and active channels, respectively. As can be seen, the mean number of idle nodes in the channel map is virtually identical to the true number of such channels, which is $(1-\gamma) N$. Mean sensing intervals for idle and active nodes are the same under uniform sensing, but differ under differential sensing, as could be expected: the presence of guard sensing nodes leads to a decrease of mean sensing interval for idle nodes. The reduction is particularly noticeable at values of duty cycle above, say, 0.6 or so. Varying the number of guard sensors can lead to further improvement, albeit at a smaller scale. In both cases, the price to pay is an increase in the mean sensing interval for active nodes.
The errors in the sensing process, in terms of both the number of channels for which erroneous information is recorded in the channel map and the delay in detecting a change in activity on the channel, are shown in Figs. 4 and 5, respectively. The error under uniform sensing is mostly dependent on the number of channels, and decreases only slightly towards the edges of the range of duty cycles observed; this is due to the reduction in number of nodes in these ranges - note that, when $N=50$, the mean number of idle and active nodes at $\gamma=0.9$ is 5 and 45 , respectively, and the changes occur less frequently than in the middle of the range of $\gamma$ values.

Under differential sensing, for variable $N$ and $\gamma$, the error in the number of active channels has a similar shape, but it is about twice as large. The error in the number of idle channels, however, peaks at about $75 \%$ of the error under uniform sensing, and rapidly decreases when the number of channels decrease and/or the duty 


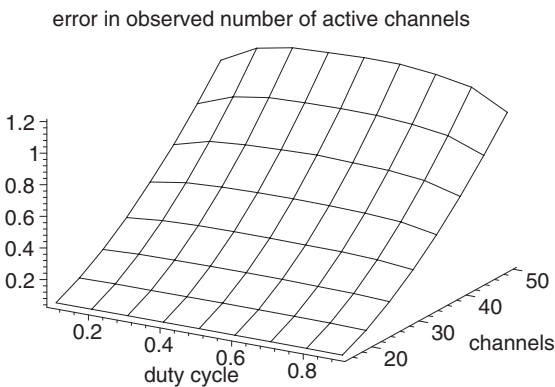

(a) Mean error in the number of idle channels thought to be active under uniform random sensing, $N$ and $\gamma$ variable.

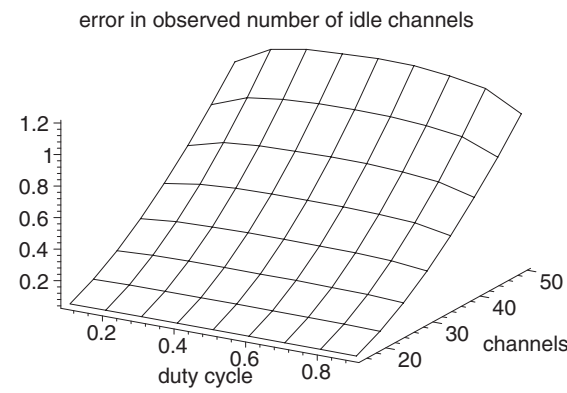

(d) Mean error in the number of active channels thought to be idle under uniform random sensing, $N$ and $\gamma$ variable.

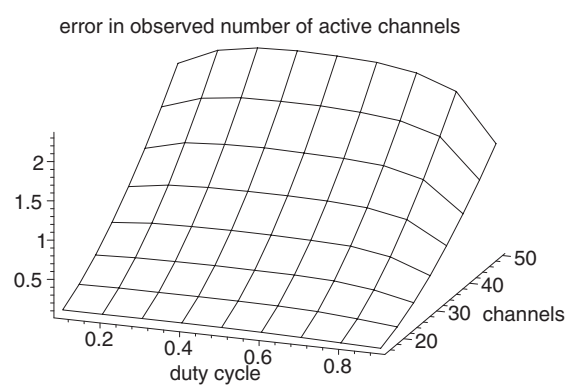

(b) Mean error in the number of idle channels thought to be active under split sensing, $N$ and $\gamma$ variable.

error in observed number of idle channels

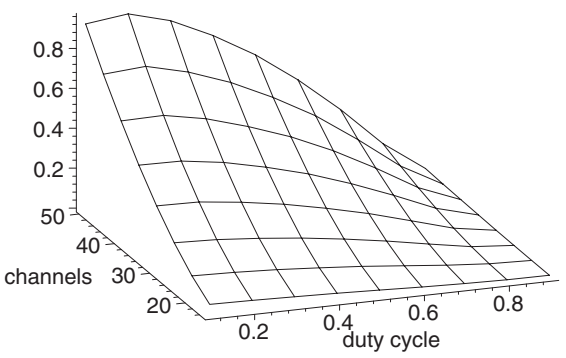

(e) Mean error in the number of active channels thought to be idle under split sensing, $N$ and $\gamma$ variable.

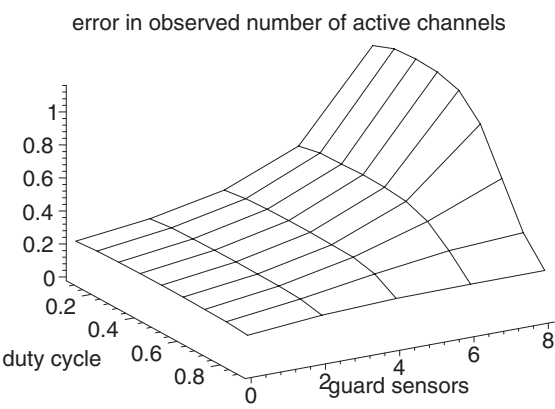

(c) Mean error in the number of idle channels thought to be active under split sensing, $\gamma$ and $X_{i}$ variable.

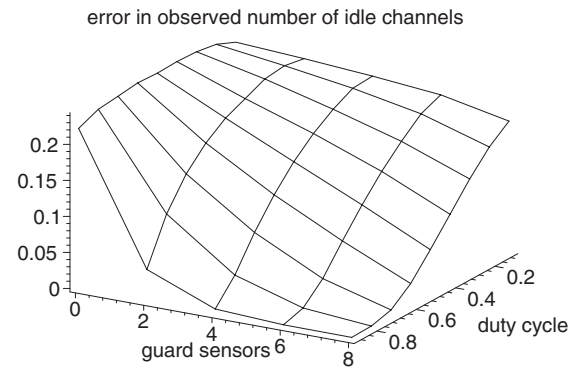

(f) Mean error in the number of active channels thought to be idle under split sensing, $\gamma$ and $X_{i}$ variable.

Figure 4: Sensing error magnitude.

cycle increases. This may be explained by the improved ratio of channels to sensor nodes and the reduction in the mean number of active channels. For example, under $\gamma=0.9$ and $N=30$, there are only three idle channels on the average; since $X_{i}=5$, each idle channels is sensed in virtually every sensing cycle.

Increasing the number of guard nodes leads to further reductions in error magnitude, but again the improvement applies to idle channels only.

Regarding detection delays (which are more interesting), the introduction of differential sensing leads to marked improvements in comparison with uniform sensing, esp. with respect to delays for beginning of channel activity. Under differential sensing with $X_{i}>4$, this delay falls to around $0.5 T_{s}$ which is close to the theoretical minimum.

Our other hypothesis, namely, that the improvement of sensing accuracy may be obtained by reducing the number of working channels, is also vividly confirmed through the diagrams in Figs. 4 and 5. With $X=10$, substantial reductions in error magnitude and duration may be obtained by going from $N=50$ down to 30 or even lower. The choice of channels to retain in the working set, however, critically depends on activity patterns of primary users. Learning the dynamics of primary user activity and adapting the operation of the CPAN accordingly remains one of the promising areas for future research.

Obviously, the analytical framework presented in this paper may be used to design cognitive piconets with a given threshold for sensing error, be it through the number of channels or the detection delay, or maybe even both. In case the number of channels is fixed, it is possible to analytically determine the minimum number of sensing nodes that would bring the error below those thresholds.
If the number of sensing nodes is fixed, the desired accuracy may be obtained by reducing the number of channels in the working set and/or adjusting the number of sensing nodes in the guard set. The exact procedure for the optimum choice of network parameters, given the performance target values, remains to be addressed in our future work.

We note that a number of papers deal with sensing issues in cognitive networks $[15,10,13,17]$. However, most of them discuss sensing from the perspective of the physical (PHY) layer of the protocol stack, and thus take 'cooperation' to mean the combination of sensing results from several sensing nodes in order to improve the confidence in those results, rather than in the sense used in this paper.

\section{CONCLUSION}

Analytical results presented here allow us to conclude that accurate channel sensing is feasible even when the number of sensing nodes is well below the number of available channels. Furthermore, differential sensing may help reduce the sensing error, expressed through the number of idle channels for which the information in the channel map is incorrect and/or the delay in detecting the beginning of primary user activity on the channels from the working set, to almost any desired value; the price to pay for this improvement is the decrease in accuracy with respect to active channels.

In our future work, we plan to address imperfect transmission of sensing results, learning and adapting to the dynamics of primary users, and the design, analysis, and optimization of a MAC protocol suitable for frequency-hopping CPANs. 
detection delay for beginning of activity

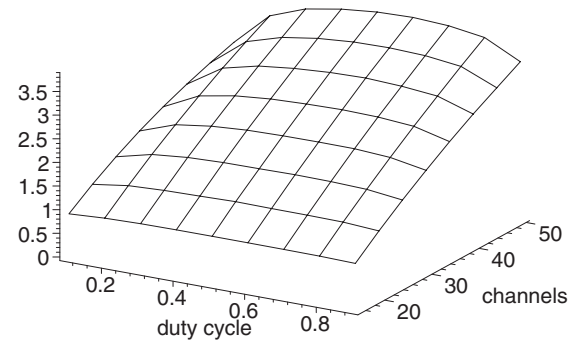

(a) Delay in detecting the beginning of channel activity under uniform random sensing, $N$ and $\gamma$ variable.

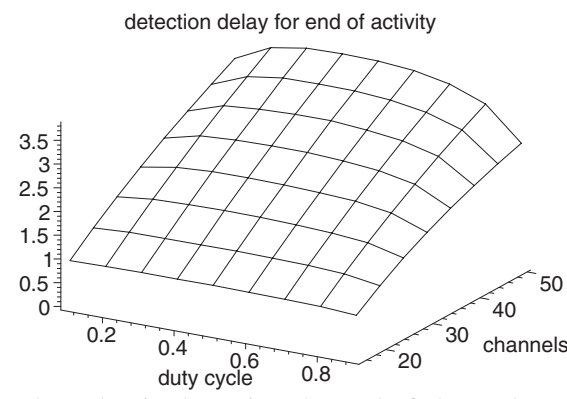

(d) Delay in detecting the end of channel activity under uniform random sensing, $N$ and $\gamma$ variable.

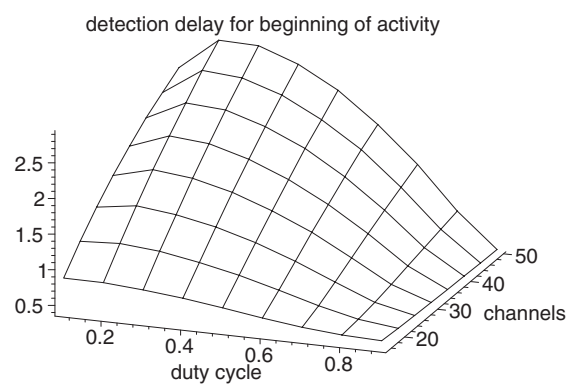

(b) Delay in detecting the beginning of channel activity under sensing, $N$ and $\gamma$ variable.

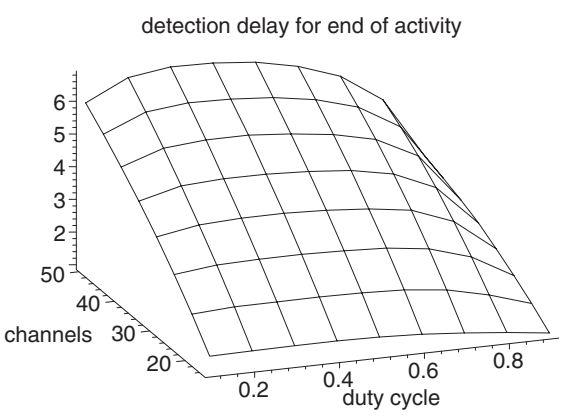

(e) Delay in detecting the end of channel activity under sensing, $N$ and $\gamma$ variable.

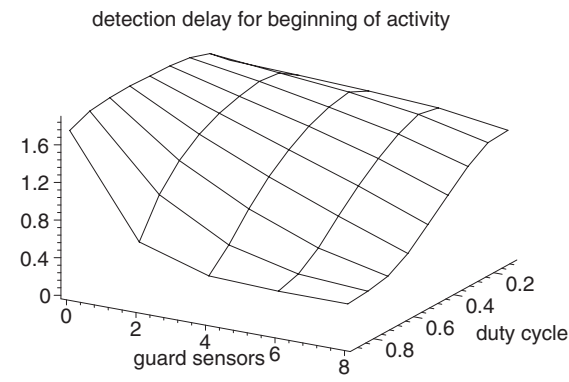

(c) Delay in detecting the beginning of channel activity under split sensing, $\gamma$ and $X_{i}$ variable.

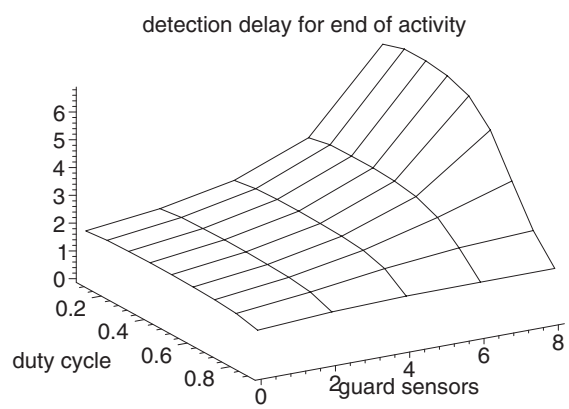

(f) Delay in detecting the end of channel activity under split sensing, $\gamma$ and $X_{i}$ variable.

Figure 5: Sensing error duration.

\section{REFERENCES}

[1] I. F. Akyildiz, W.-Y. Lee, M. C. Vuran, and S. Mohanty. $\mathrm{NeXt}$ generation/dynamic spectrum access/cognitive radio wireless networks: A survey. Computer Networks, 50:2127-2159, 2006.

[2] Bluetooth SIG. Core Specification of the Bluetooth System. Version 2.0 + EDR, Nov. 2004.

[3] C.-T. Chou, N. Sai Shankar, H. Kim, and K. G. Shin. What and how much to gain by spectrum agility? IEEE Journal on Special Areas in Communications - Wireless Series, 25(3):576-588, Apr. 2007.

[4] S. Geirhofer, L. Tong, and B. M. Sadler. Cognitive medium access: A protocol for enhancing coexistence in WLAN bands. In Proceedings Global Telecommunications Conference GLOBECOM'07, Washington, DC, Nov. 2007.

[5] D. P. Heyman and M. J. Sobel. Stochastic Models in Operations Research, Volume I: Stochastic Processes and Operating Characteristics. McGraw-Hill, New York, 1982.

[6] W. Hu and E. Sofer. IEEE 802.22 wireless RAN standard PHY and MAC proposal. technical proposal submitted to IEEE 802.22 WG, 22-05-0098-00-0000, IEEE 802.22 WG, 2005.

[7] IEEE. Wireless MAC and PHY specifications for high rate WPAN. IEEE Std 802.15.3, IEEE, New York, NY, 2003.

[8] P. K. Lee. Joint frequency hopping and adaptive spectrum exploitation. In IEEE Military Communications Conference MILCOM2001, volume 1, pages 566-570, Oct. 2001.

[9] Maplesoft, Inc. Maple 11. Waterloo, ON, Canada, 2007.

[10] S. Mishra, A. Sahai, and R. Brodersen. Cooperative sensing among cognitive radios. In Proceedings of IEEE
International Conference on Communications ICC 2006 , volume 4, pages 1658-1663, Istanbul, Turkey, June 2006.

[11] J. Mišić and V. B. Mišić. The impact of inaccurate sensing information in cognitive wireless personal area networks. In IEEE CogNet 2008, Beijing, China, May 2008.

[12] V. B. Mišić and J. Mišić. Performance of incomplete channel sensing for frequency hopping cognitive personal area networks. In WWASN2008, Beijing, China, June 2008.

[13] Z. Quan, S. Cui, and A. H. Sayed. An optimal strategy for cooperative spectrum sensing in cognitive radio networks. In Proceedings Global Telecommunications Conference GLOBECOM'07, pages 2947-2951, Washington, DC, Nov. 2007.

[14] H. Takagi. Queueing Analysis, volume 1: Vacation and Priority Systems. North-Holland, Amsterdam, The Netherlands, 1991.

[15] D. Čabrić, S. M. Mishra, D. Willkomm, R. Brodersen, and A. Wolisz. A cognitive radio approach for usage of virtual unlicensed spectrum. In Proc. 14th IST Mobile Wireless Communications Summit, Dresden, Germany, June 2005.

[16] Z. Yang and J. J. Garcia-Luna-Aceves. Hop-reservation multiple access (HRMA) for ad-hoc networks. In Eighteenth Annual Joint Conference of the IEEE Computer and Communications Societies INFOCOM'99, volume 1, pages 194-201, New York, NY, Mar. 1999.

[17] J. Zhao, H. Zheng, and G.-H. Yang. Spectrum sharing through distributed coordination in dynamic spectrum access networks. Wireless Communications and Mobile Computing, in press. 